\title{
Paper \\ A Soft-Output STBC Decoder for Aeronautical Telemetry
}

\author{
Yves Louët ${ }^{1}$, Rami Othman ${ }^{1,2}$, and Alexandre Skrzypczak ${ }^{2}$ \\ ${ }^{1}$ IETR/CentraleSupélec, Cesson-Sévigné, France \\ ${ }^{2}$ Zodiac Data Systems, Colombelles, France
}

https://doi.org/10.26636/jtit.2020.138319

\begin{abstract}
Alamouti encoding is a well-known space time block encoding technique used to improve the received signal quality in Rayleigh fading channels. In aeronautical telemetry, this encoding technique is applied to shaped offset quadrature phase shift keying tier generation (SOQPSK-TG) modulation in order to handle the two-antenna issue. It is provided for in telemetry-related IRIG standards. In this paper, we propose a unique decoding architecture for Alamouti-encoded SOQPSK-TG signals, taking advantage of pulse amplitude modulation decomposition with soft and hard outputs. We exploit this result to obtain a Viterbi algorithm (VA) for hard decoding and a soft output Viterbi algorithm (SOVA) for soft and hard decoding, with a twofold benefit: operation using one trellis structure, unlike decoders that are based on the 8-waveforms cross-correlated trellis-coded quadrature modulation (XTCQM) approximation, and very attractive bit error rate performance, as well as a complexity trade-off.
\end{abstract}

Keywords-continuous phase modulation (CPM), pulse amplitude modulation (PAM), shaped offset quadrature phase shift keying (SOQPSK), space time block coding (STBC), ternary symbol.

\section{Introduction}

In recent years, the increased demand for high data rates, despite a limited bandwidth available in aeronautical telemetry, has driven the IRIG standardization committee [1] to adopt new bandwidth efficient modulations. Shaped offset quadrature phase shift keying tier generation (SOQPSK-TG) is one of these modulations and is currently replacing the legacy solution, i.e. pulse code modulation/frequency modulation (PCM/FM). It is a continuous phase modulation (CPM) technique characterized by ternary symbols and a longer frequency pulse compared with the historic PCM/FM approach.

In order to guarantee an omnidirectional transmission from an aircraft, two SOQPSK-TG signals are sent to a ground base station. If the same signals are transmitted simultaneously, the received signal could suffer from severe interference due to the considerable phase angle differences between them. This phenomenon is known in aeronautical telemetry [2] as the "two-antenna problem". Jensen et al. showed, in [3], that by applying Alamouti encoding [4], this problem is overcome without the expansion of the re- quired bandwidth. This encoding technique was originally developed for MPSK-type modulations to improve the received signal quality while keeping the receiver side processing simple. However, application of this idea in practical aeronautical telemetry systems is not obvious, as emphasized in [2]. This is essentially due to the peculiar nature of aeronautical telemetry modulation (SOQPSK-TG), as described above, and to the fact that the signals are received at each antenna with different delays. The difference in delays is relatively high and thus it should be estimated along with channel gains and frequency offset before decoding.

In this paper, we focus on decoding algorithms and we assume that the necessary parameters listed above are perfectly estimated using the estimation algorithms introduced in [2] for Alamouti encoded SOQPSK-TG signals. Several decoding algorithms have been explored in [5], [6] taking into account the CPM-like nature of SOQPSK-TG at first and then the presence of differential delays. The decoder that offers the best bit error rate performance and the highest complexity trade-off relies on the least squares principle whose decision metric is a function of the quadratic error between the received signal samples and their noiseless estimates. The latter are built using the eight waveform crosscorrelated trellis-coded quadrature modulation (XTCQM) approximation of SOQPSK-TG in order to maintain a reasonable level of complexity. The resulting algorithm provides good BER performance, how-ever it operates with two different trellis structures depending on the sign of the differential delays. Moreover, the output of this decoder does not provide the log likelihood ratios (LLR) of the decoded bits and, therefore, we cannot fully take advantage of the performance improvement offered by the presence of forward error correction (FEC) systems [7].

In order to be able to do so, we reformulate the Alamouti decoding problem using pulse amplitude modulation (PAM) approximations of SOQPSK-TG [8] instead of the XTCQM approximation [9]. We consider that SOQPSKTG can be approximated as a linear modulation or as a combination of two linear modulations whose waveforms and pseudo-symbols are described in [8]. We show that using these PAM approximations allows us to build a single trellis structure, regardless of the sign of the differential 
delay. The proposed trellis is less complex than the ones proposed in [5]. We then exploit this result to get a Viterbi algorithm (VA) for hard decoding, and a soft output Viterbi algorithm (SOVA) inspired from [10] for soft and hard decoding. These decoders may operate using both first and the second PAM approximations. We also propose a further complexity reduction by associating the proposed decoder with the $M$-algorithm [11]. This allows to reduce the number of the computed branches of the trellis, as well as the number of comparators. By combining SOVA with the $M$-algorithm, a new architecture is created (denoted as M-SOVA in this paper), differing from the soft output M-algorithm (SOMA) [12], as the latter is based on conventional SOVA, as detailed in [13]. Finally, we present performance-related results of the different decoders, with and without channel coding. We show that the decoder that uses the second PAM approximation performs better than the decoder from [5] and is more robust in terms of differential delays. We also highlight that the combination of the proposed M-SOVA for Alamouti-encoded SOQPSKTG with IRIG normalized channel encoders [1] offers very attractive BER performance.

This paper is organized as follows. The signal model is described in Section 2. The problem formulation is summarized in Section 3. A detailed description of the proposed Alamouti decoders is provided in Section 4 and their complexities to the least squares XTCQM Alamouti decoder are compared. Finally, in Section 5 the BER performance of the decoders for different scenarios is given.

\section{Signal Model}

\subsection{CPM Definition of SOQPSK-TG}

The complex envelope of SOQPSK is expressed as [14]:

$$
s(t ; \bar{\alpha})=\sqrt{\frac{E_{s}}{T}} \mathrm{e}^{\mathrm{j} \sum_{n} \alpha_{n} q(t-n T)},
$$

where $E_{s}$ is the energy per information symbol, $T$ is the symbol time duration and $\bar{\alpha}=\left\{\alpha_{n}\right\}_{n \in \mathbb{Z}}$ are ternary symbols from the alphabet $\{-1,0,+1\}$ which are generated according to the following mapping principle:

$$
\alpha_{n}=(-1)^{n+1} \frac{b_{n-1}\left(b_{n}-b_{n-2}\right)}{2},
$$

where $\left\{b_{n}\right\}_{n \in \mathbb{N}} \in\{-1,+1\}$. Function $q(t)$, namely the phase pulse, is the time integral of the frequency pulse $g(t)$ whose time support is equal to $L T$ and is weighted by modulation index $h$. The frequency pulse of SOQPSK-TG is of length $L=8$ and is described in [1].

\subsection{PAM Decomposition of SOQPSK-TG}

The fact that the transmitted ternary symbols of SOQPSK are encapsulated in the instantaneous frequency makes the handling of this modulation less tractable. Thus, several mathematical representations and approximations were proposed in the literature, such as the (XTCQM) representation [15] and the PAM decomposition [8], [16]. In this paper, we focus on PAM decomposition introduced in [8], where it has been shown that SOQPSK-TG may accurately be approximated by two linear modulations at the most, i.e. $s(t)$ may be written as:

$$
s(t) \approx \sum_{j=0}^{1} \sum_{i} \rho_{j, i} w_{i}(t-i T)
$$

or

$$
s(t) \approx \sum_{i} \rho_{0, i} w_{i}(t-i T)
$$

where

$$
\left\{\begin{array}{lll}
\rho_{0, i}=b_{i}, & \rho_{1, i}=-b_{i-1} b_{i} b_{i+1}, & i \text { even, } \\
\rho_{0, i}=j b_{i}, & \rho_{1, i}=-j b_{i-1} b_{i} b_{i+1}, & i \text { odd. }
\end{array}\right.
$$

Functions $\left\{w_{i}(t)\right\}_{i \in\{0,1\}}$ are plotted in Fig. 1 [8].

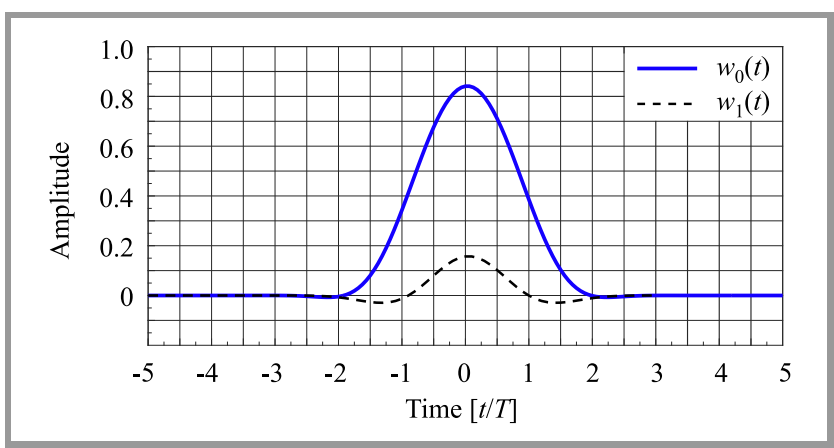

Fig. 1. PAM representation of SOQPSK-TG using [8].

Based on Eq. (3) and Fig. 1, the sampled version of SOQPSK-TG may be approximated as:

$$
s(n T) \approx \sum_{k=-1}^{+1} \rho_{0, n-k} w_{0}(k T)+\rho_{1, n} w_{1}(0),
$$

since we can suppose that:

$$
\begin{cases}w_{0}(n T)=0, & \text { if } \quad|n| \geq 2 \\ w_{1}(n T)=0, & \text { if } \quad|n| \geq 1 .\end{cases}
$$

Similarly, if we take into account the approximation described in Eq. (4), we can simply consider that $w_{1}(0)=0$ in Eq. (6).

\subsection{Alamouti Space-Time Coding for SOQPSK-TG}

In aeronautical telemetry, Alamouti STBC is becoming an attractive solution to create space diversity and to combat the "two-antenna problem" without expanding the received signal bandwidth [3]. It consists in encoding the bit stream $\underline{b}=\left[b_{i}\right]_{i \in \mathbb{N}}$ into two parallel bits streams $\left(\underline{b}^{0}, \underline{b}^{1}\right)$ in the following manner:

$$
\begin{aligned}
& \underline{b}^{0}=\left[\ldots \quad b_{4 n}, b_{4 n+1},-b_{4 n+2}, b_{4 n+3}, \quad \ldots\right], \\
& \underline{b}^{1}=\left[\ldots \quad b_{4 n+2}, b_{4 n+3}, b_{4 n},-b_{4 n+1}, \quad \ldots\right] \text {. }
\end{aligned}
$$


Each encoded bit stream is then passed through an SOQPSK-TG modulator and transmitted over an antenna with the same carrier frequency.

\section{Problem Formulation}

In this paper, we consider the MISO case, i.e. the receiver is made up of one antenna only. Thus, the received signal model is the following

$$
r(t)=\left[h_{0} s_{0}(t)+h_{1} s_{1}(t-\Delta \varepsilon)\right] \mathrm{e}^{j 2 \pi f_{0} t}+u(t),
$$

where $s_{0}(t)$ and $s_{1}(t)$ are two SOQPSK-TG signals containing the bit streams $\underline{b}^{0}$ and $\underline{b}^{1}$ and are affected by complexvalue attenuations $\bar{h}_{0}$ and $\bar{h}_{1}$, respectively. Parameter $\Delta \varepsilon$ is a differential delay which is generally considered in aeronautical telemetry [2], $f_{0}$ is the frequency offset and $u(t)$ is an additive complex white Gaussian noise (AWGN). The received signal is filtered to reduce the noise level and sampled at the bit rate at $t=n T$ and $t=n T+\Delta \varepsilon$. We suppose that $f_{0}$ is null, therefore the sampled signal $r^{f}$ can be written as:

$$
\left\{\begin{array}{c}
r^{f}(n T)=h_{0} s_{0}^{f}(n T)+h_{1} s_{1}^{f}[(n-\Delta \tau) T]+u^{f}(n T), \\
r^{f}(n T+\Delta \varepsilon)=h_{0} s_{0}^{f}[(n+\Delta \tau) T]+h_{1} s_{1}^{f}(n T) \\
+u^{f}[(n+\Delta \tau) T]
\end{array}\right.
$$

where $r^{f}, s_{0}^{f}, s_{1}^{f}$ and $u_{0}^{f}$ are the filtered versions of $r, s_{0}, s_{1}$ and $u_{0}$, respectively. Parameter $\Delta \tau=\frac{\Delta \varepsilon}{T}$ is the differential delay relatively to the sample time and it is assumed that $|\Delta \tau|<1$.

The aim of the decoder is to recover the bit stream $\underline{b}$ from $r^{f}(n T)$. To do so, it is necessary to estimate the channel parameters and then to feed them to the decoder. In the remaining sections of the paper, we assume that these parameters are perfectly estimated using the estimators described in [2] and are denoted by $\hat{h}_{0}, \hat{h}_{1}$ and $\Delta \hat{\tau}$. Thus, the maximum likelihood (ML) of $\underline{b}$ performs:

$$
\underline{\hat{b}}=\underset{b}{\operatorname{argmin}}\left\{\Lambda(\underline{b})+\Lambda_{\Delta \hat{\tau}}(\underline{b})\right\}
$$

where

$$
\Lambda(\underline{b})=\sum_{n=-\infty}^{+\infty} \mid r^{f}(n T)-\left[\hat{h}_{0} s_{0}^{f}(n T)+\left.\hat{h}_{1} s_{1}^{f}[(n-\Delta \hat{\tau} T)]\right|^{2}\right.
$$

and

$$
\Lambda_{\Delta \hat{\tau}}(\underline{b})=\sum_{n=-\infty}^{+\infty}\left|r^{f}(n T+\Delta \hat{\tau})-\left[\hat{h}_{0} s_{0}^{f}(n T+\Delta \hat{\tau})+\hat{h}_{1} s_{1}^{f}(n T)\right]\right|^{2}
$$

The log-likelihood functions $\Lambda(\underline{b})$ and $\Lambda_{\Delta \hat{\tau}}(\underline{b})$ represent the error between the received samples and a reconstructed version of the noiseless signal. The complexity of the decoder depends on the memory effect introduced by the CPM nature of SOQPSK-TG. If we use the original definition of
SOQPSK-TG, as presented in Eq. (1), each $\left\{s_{i}(n T)_{i \in\{0,1\}}\right\}$ depends on 11 consecutive symbols [15], which makes the decoding process highly complex, since the VA would operate with at least 2048 states. In order to overcome this, the authors in [5] took advantage of the XTCQM approximation to derive a least square sequence hard decoder with two trellis structures which depend on the sign of the differential delay $\Delta \hat{\tau}$. In the next section, we propose a unique trellis structure that does not depend on the sign of $\Delta \hat{\tau}$ and we apply a VA whose branch metrics rely on the PAM approximations of Eqs. (3) and (4). We then adapt the proposed solution to get soft outputs via the SOVA introduced in [10], and we introduce a more reduced complexity architecture thanks to the $M$-algorithm [11], namely the M-SOVA.

\section{Proposed MLSE Decoder}

\subsection{Proposed Viterbi Algorithm}

In order to resolve Eq. (12), we define an equivalent symbol $S_{n}$ as the concatenation of the 4 consecutive bits which come into play in the encoding process, i.e. $S_{n}=$ $\left[\tilde{b}_{4 n}, \tilde{b}_{4 n+1}, \tilde{b}_{4 n+2}, \tilde{b}_{4 n+3}\right]$, such that $\tilde{b}_{4 n}=\frac{1}{2}\left(b_{4 n}+1\right)$. The ML estimate of Eq. (12) is equivalent to:

$$
\underline{\hat{S}}=\underset{S}{\operatorname{argmin}}\left\{\Lambda(\underline{S})+\Lambda_{\Delta \hat{\tau}}(\underline{S})\right\}
$$

where

$$
\begin{aligned}
\Lambda(\underline{S}) & =\sum_{n=-\infty}^{+\infty}\left(\sum_{k=-1}^{2} \mid r^{f}(4 n T+k T)-\left[\hat{h}_{0} s_{0}^{f}(4 n T+k T)\right.\right. \\
& \left.+\left.\hat{h}_{1} s_{1}^{f}[(4 n-\Delta \hat{\tau}) T+k T]\right|^{2}\right), \\
& =\sum_{n=-\infty}^{+\infty} \Lambda(n),
\end{aligned}
$$

and

$$
\begin{aligned}
\Lambda_{\Delta \hat{\tau}}(\underline{S}) & =\sum_{n=-\infty}^{+\infty}\left(\sum_{k=-1}^{2} \mid r^{f}(4 n T+k T+\Delta \hat{\tau})\right. \\
& \left.-\left.\left[\hat{h}_{0} s_{0}^{f}(4 n T+k T+\Delta \hat{\tau})+\hat{h}_{1} s_{1}^{f}(4 n T+k T)\right]\right|^{2}\right) \\
& =\sum_{n=-\infty}^{+\infty} \Lambda_{\Delta \hat{\tau}}(n)
\end{aligned}
$$

Formulating the problem as in Eq. (15) allows us to apply the maximum likelihood sequence estimation (MLSE) VA to estimate the equivalent symbol sequence $\underline{S}$. The trellis of VA is given in Fig. 2 and is composed of 16 states and 256 branches. Let the state of the node $i$ at the epoch $n$ be denoted by $S_{n}(i)$. Using Eqs. (6), (17) and (19), the 


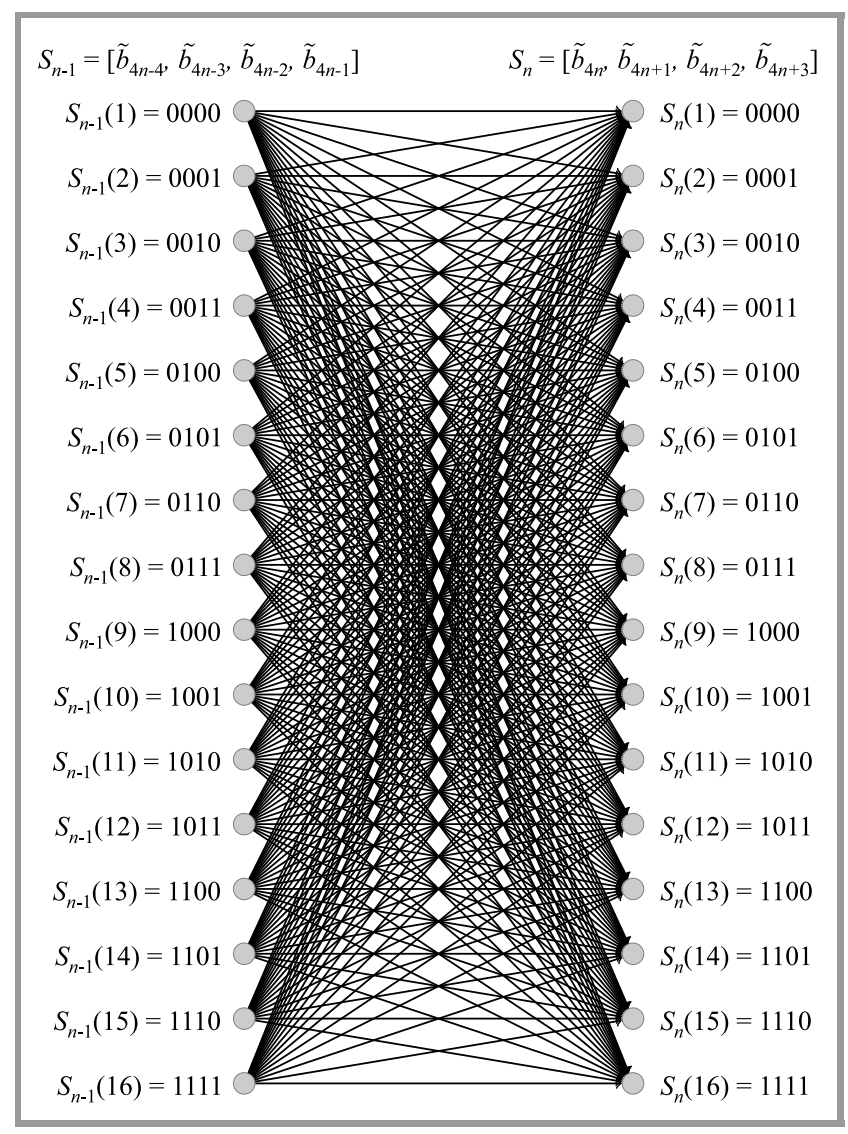

Fig. 2. Trellis of the proposed decoder.

branch metric associated to the transition $S_{n-1}(i) \rightarrow S_{n}(j)$ is defined as:

$$
\begin{aligned}
\lambda\left[S_{n-1}(i), S_{n}(j)\right] & =\Lambda(n)+\Lambda_{\Delta \hat{\tau}}(n), \\
& =\sum_{k=-1}^{2}\left|B_{k, 0}\right|^{2}+\left|B_{k, \Delta \hat{\tau}}\right|^{2},
\end{aligned}
$$

where

$$
\begin{gathered}
B_{k, 0}=r^{f}[(4 n+k) T]-\hat{h}_{0}\left(\sum_{i=-1}^{1} \rho_{0,4 n+k-i}^{(0)} w_{0}^{f}(i T)\right. \\
\left.+\rho_{1,4 n+k}^{(0)} w_{1}^{f}(0)\right)-\hat{h}_{1}\left(\sum_{i=-1}^{1} \rho_{0,4 n+k-i}^{(1)} w_{0}^{f}[(i-\Delta \hat{\tau}) T]\right. \\
\left.+\rho_{1,4 n+k}^{(1)} w_{1}^{f}(-\Delta \hat{\tau} T)\right),
\end{gathered}
$$

and

$$
\begin{aligned}
& B_{k, \Delta \hat{\tau}}=r^{f}[(4 n+k+\Delta \hat{\tau}) T] \\
& \left.-\hat{h}_{0}\left(\sum_{i=-1}^{1} \rho_{0,4 n+k-i}^{(0)} w_{0}^{f}[i+\Delta \hat{\tau}) T\right]+\rho_{1,4 n+k}^{(0)} w_{1}^{f}(\Delta \hat{\tau})\right) \\
& \quad-\hat{h}_{1}\left(\sum_{i=-1}^{1} \rho_{0,4 n+k-i}^{(1)} w_{0}^{f}(i T)+\rho_{1,4 n+k}^{(1)} w_{1}^{f}(0)\right)
\end{aligned}
$$

The sets of pseudo-symbols $\left(\rho_{0, k}^{(i)}, \rho_{1, k}^{(i)}\right)$ are associated with signal $s_{i}(t)$ and the pulses $w_{i}^{f}$ are the filtered versions of $w_{i}$, where $i \in\{0,1\}$. The filtered version of $w_{i}$ is very similar to $w_{i}$ and, therefore, it makes the approximation of (6) valid for $w_{i}^{f}$ as well. Both Eqs. (22) and (23) are valid regardless of the sign of $\Delta \hat{\tau}$, unlike in the sub-metrics described in [5]. This is due to the use of the PAM approximation instead of XTCQM. A more detailed discussion regarding the proposed branch metrics is presented in Subsection 4.3. Once the branch metrics $\lambda\left[S_{n-1}(i), S_{n}(j)\right]$ are calculated, the cumulative metrics $\Gamma_{n}\left[S_{n}(j)\right]$ can be updated as follows:

$$
\Gamma_{n}\left[S_{n}(j)\right]=\min _{i}\left[\gamma_{n}\left(S_{n-1}(i), S_{n}(j)\right)\right],(i, j) \in\{1, \ldots, 16\}^{2},
$$

where

$$
\gamma_{n}\left[S_{n-1}(i), S_{n}(j)\right]=\Gamma_{n-1}\left[S_{n-1}(i)\right]+\lambda\left[S_{n-1}(i), S_{n}(j)\right] .
$$

Once the cumulative metrics are calculated and the survivor paths are stored for a given depth $\delta$, we perform a traceback loop to obtain $\underline{\hat{S}}$ and, therefore, the underlying bit sequence $\underline{\hat{b}}$.

\subsection{Soft Decision Decoder}

Here, we take advantage of the VA to obtain soft decisions instead of hard ones. To do so, we apply the MAX-LogMAP equivalent SOVA for non-binary codes, as introduced in [10]. This solution is well suited to the presented case, since the proposed trellis is built on the equivalent nonbinary symbol $S_{n}$ and since the branch metrics introduced in Eq. (21) represent the forward recursion of SOVA thanks to the elegant interpretation of the MAX-LOG-MAP algorithm given in [17]. Based on [13] and instead of storing the survivor paths, we rather store the reliability difference at epoch $n$ whose definition is:

$$
\begin{array}{r}
R_{n}\left[S_{n-1}(i), S_{n}(j)\right]=\Gamma_{n}\left[S_{n}(j)\right]-\gamma_{n}\left[\left(S_{n-1}(i), S_{n}(j)\right],\right. \\
(i, j) \in(\{1, \ldots, 16\})^{2} .
\end{array}
$$

The reliability difference of the survivor path is then equal to zero in our case. Once Eq. (26) is computed for a given depth, the backward recursion is yielded and the max-log joint probability is obtained as follows:

$$
P\left[S_{n-1}(i), S_{n}(j), r^{f}\right]=\beta_{n}\left[S_{n}(i)\right]+R_{n}\left[S_{n-1}(i), S_{n}(j)\right],
$$

where

$$
\beta_{n-1}\left(S_{n-1}(j)\right)=\min _{i}\left[R_{n}\left(S_{n-1}(i), S_{n}(j)\right)+\beta_{n}\left(S_{n}(i)\right)\right] .
$$

Finally the soft output of the symbol $S_{n}$ is:

$$
P\left(\hat{S}_{n}=\frac{S_{n}(j)}{r^{f}}\right)=\min _{i}\left[P\left(S_{n-1}(i), S_{n}(j), r^{f}\right)\right] .
$$

Since $S_{n}$ is composed of the 4 bits $\left[\tilde{b}_{4 n}, \tilde{b}_{4 n+1}, \tilde{b}_{4 n+2}, \tilde{b}_{4 n+3}\right]$, the conversion of the soft output (LLR) to the bit level is straightforward. 


\subsection{Optimizing Computational Complexity}

\subsubsection{The Branch Metrics Computation}

Equation (21) shows that the proposed branch metric is a sum of 8 sub-metrics $B_{k, 0}$ and $B_{k, \Delta \hat{\imath}}$ and, therefore, one may assume that it is necessary to compute 2,048 different values of sub-metrics in order to get Eq. (21). Fortunately, each sub-metric does not involve all the underlying bits that make up the trellis of Fig. 2. If we develop, for instance, $B_{0,0}$ :

$$
\begin{gathered}
B_{0,0}=r^{f}(4 n T)-\hat{h}_{0}\left[j b_{4 n+1} w_{0}^{f}(-T)+b_{4 n} w_{0}^{f}(0)\right. \\
\left.+j b_{4 n-1} w_{0}^{f}(T)-b_{4 n-1} b_{4 n} b_{4 n+1} w_{1}^{f}(0)\right] \\
-\hat{h}_{1}\left[j b_{4 n-3} w_{0}^{f}(-(1+\Delta \hat{\tau}) T)+b_{4 n+2} w_{0}^{f}(-\Delta \hat{\tau} T)\right] \\
\left.+j b_{4 n+3} w_{0}^{f}[(1-\Delta \hat{\tau}) T)+b_{4 n-3} b_{4 n+2} b_{4 n+3} w_{1}^{f}(-\Delta \hat{\tau} T)\right],
\end{gathered}
$$

we may notice that it only involves 6 different bits out of 8 . Thus, only 64 different values of this sub-metric are computed instead of 256. Following the same approach, it is necessary to compute only 16 different values of $B_{1, \Delta \hat{\tau}}$ since it is a function of 4 different bits. In consequence, the total number of the computed values decreases from 2,048 to 320 . The obtained result is smaller than the one based on the state-of-the-art solution [5] which is equal to 480. This optimization process does not affect the final output of Eq. (21), since the calculated values of the submetrics are simply duplicated and reordered with respect to the proposed trellis.

\subsubsection{Selected Number of Nodes and Reliability Values}

In order to estimate the underlying bit sequence and/or its soft information, the classical approach consists to make a full trellis search up to a certain depth $\delta$ and then to perform a trace-back loop. In this case, it means that it is necessary to calculate 256 branch metrics at each epoch and to store $16 \delta$ values for hard decoding or $256 \delta$ reliability values for soft decoding. In this paper, we propose to reduce the number of operations via the $M$-algorithm whose concept was introduced in [11] for hard decoding in [18] and in [12] for soft decoding. This technique is

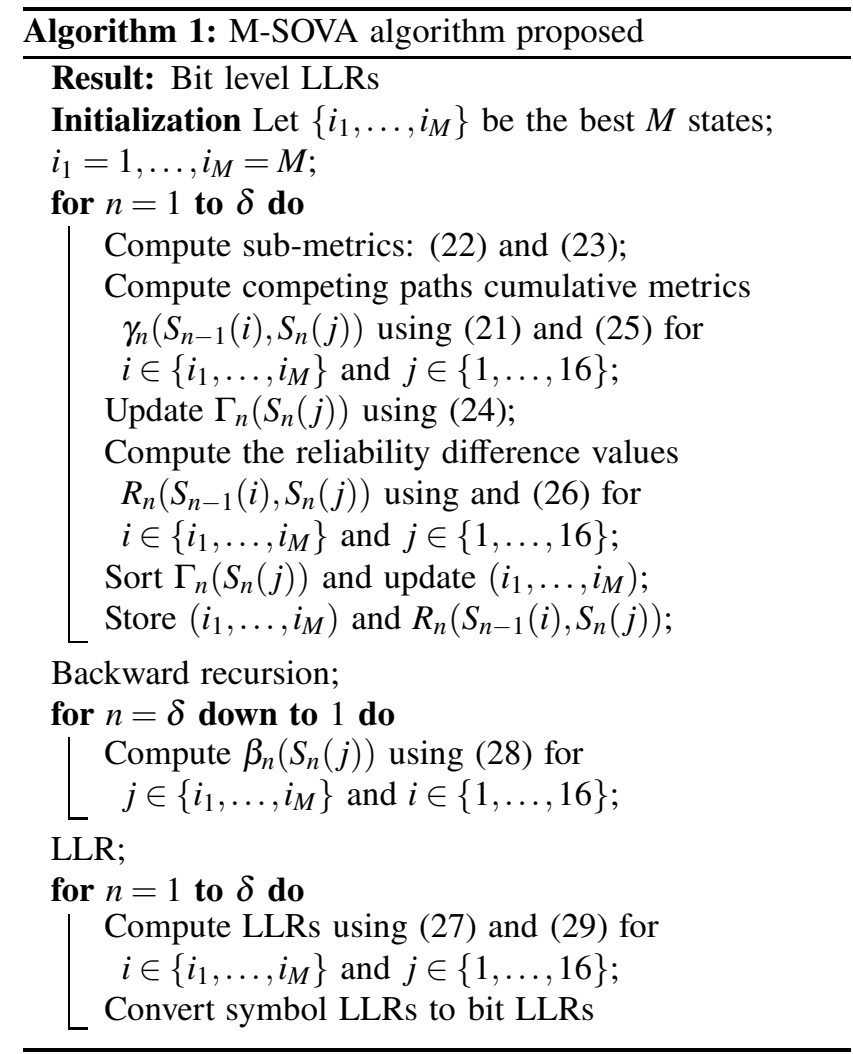

a sub-optimal tree search, as it only crosses a part of the trellis. It consists in extending the $M$ best states instead of all the states from one epoch to the next. This reduces, in the scenario presented the number of calculated branch metrics, as it decreased from 256 to $16 M$. As for soft decoding, we propose to combine the $M$-algorithm with the MAX-Log-MAP SOVA presented in Subsection 4.2, and we refer to it as M-SOVA. This approach is different from the soft output $M$-algorithm (SOMA) introduced in [12], since the latter is based on the conventional SOVA for binary codes [13]. The proposed algorithm is described in Algorithm 1, and we compare, in Table 1, the number of operations required to estimate one block of 4 bits via the state-of-the-art solution (the least squares XTCQM decoder) and the proposed algorithms (i.e. by taking a depth $\delta=1$ ). One may see that the number of

Table 1

Complexity comparison of the different decoders

\begin{tabular}{|l|c|c|c|}
\hline & State-of-the-art solution [5] & $\begin{array}{c}\text { Proposed solution } \\
\text { with Viterbi } M \text {-algorithm }\end{array}$ & $\begin{array}{c}\text { Proposed solution } \\
\text { M-SOVA }\end{array}$ \\
\hline \hline Addition & 3968 & $640+128 M$ & $644+176 M$ \\
\hline Multiplication & 960 & 640 & 640 \\
\hline$\|\cdot\|^{2}$ & 480 & 320 & 320 \\
\hline Comparison & 240 & $15 M$ & $46 M+40$ \\
\hline Sorting operation of 16 elements & 0 & 1 & 1 \\
\hline Number of stored values & 16 & 16 & $17 M$ \\
\hline
\end{tabular}


additions and multiplications is relatively high. This is mainly due to the sub-metrics computations, since they require 960 multiplications and 1,920 additions if we take the state-of-the-art solution approach [6] is adopted. On the other hand, the computation of the 8 sub-metrics involves 640 multiplications and 640 additions in our case. The use of the $M$-algorithm may further reduce the number of operations and the required storage memory, without adding a significant degree of complexity due to the use of sorting algorithms, since the latter have an average complexity order of $O[16 \log (16)]$ [12].

\section{Simulation Results}

In all of simulations, we consider that $\left|h_{0}\right|=\left|h_{1}\right|=\frac{\sqrt{2}}{2}$.

\subsection{BER Performance as a Function of the PAM Approximation}

We start by evaluating the different algorithms for uncoded systems, i.e. without applying channel coding. In Fig. 3 and Fig. 4, we plot the BER curves of the detectors for different values of $\Delta \phi=\arg \left(h_{1}\right)-\arg \left(h_{0}\right)$. In the different figures, approx. 1 refers to the computing of the branch metrics by assuming that $w_{1}^{f}(0)=0$. On the other hand, approx. 2 takes into account $w_{1}^{f}(0)$ in the branch metrics computation. It can be seen that the performance of different detectors changes along with the phase angle difference of $h_{0}$ and $h_{1}$. One may also notice that using the 2nd approximation offers the best BER performance among all the detectors. Moreover, the performance gap between the proposed algorithm with approx. 2 and the state-of-the-art solution increases when the differential delay $\Delta \tau$ increases, as shown in Fig. 5.

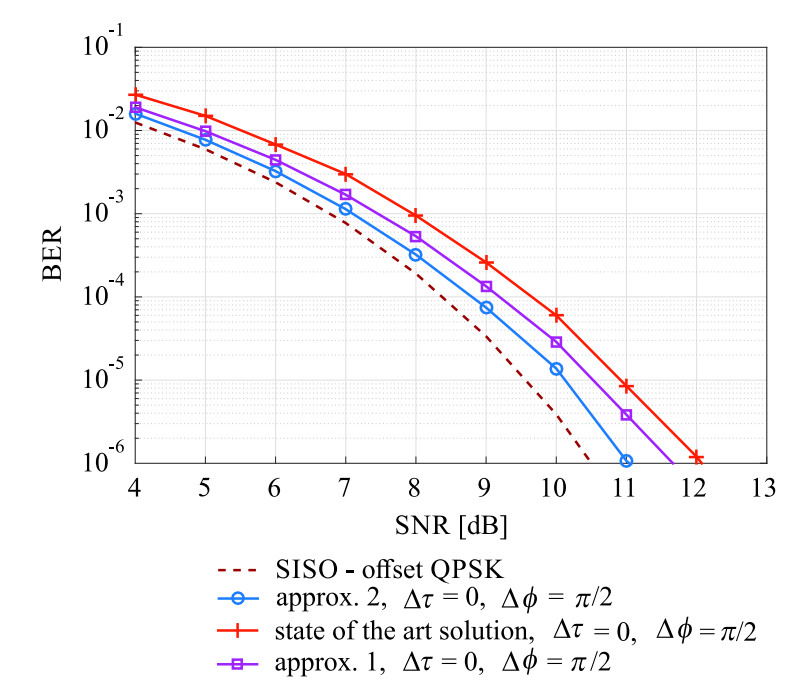

Fig. 3. BER performance for the different PAM approximations, $\Delta \tau=0, \Delta \phi=\frac{\pi}{2}$.

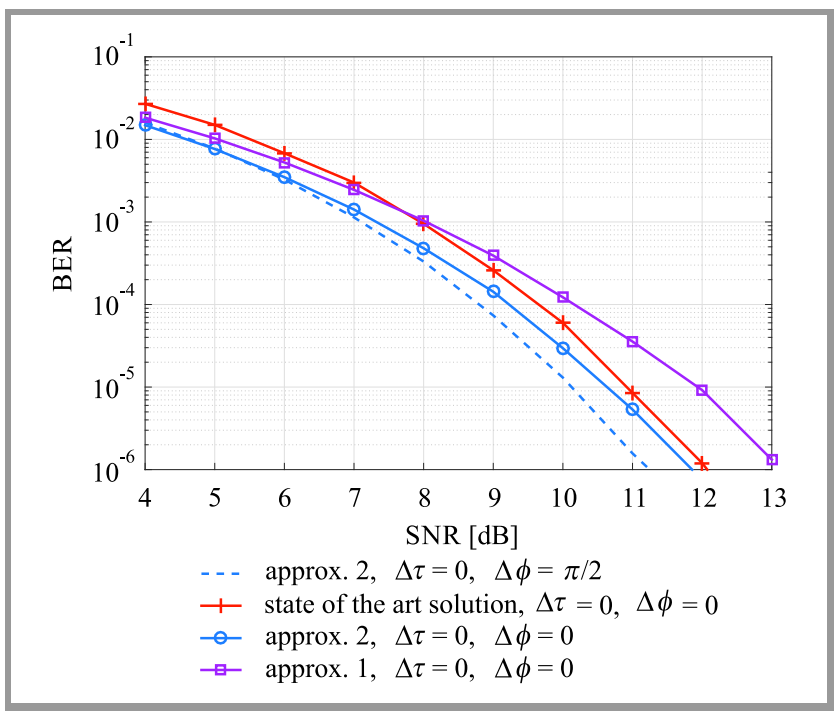

Fig. 4. BER performance for the different PAM approximations, $\Delta \tau=0, \Delta \phi=0$.

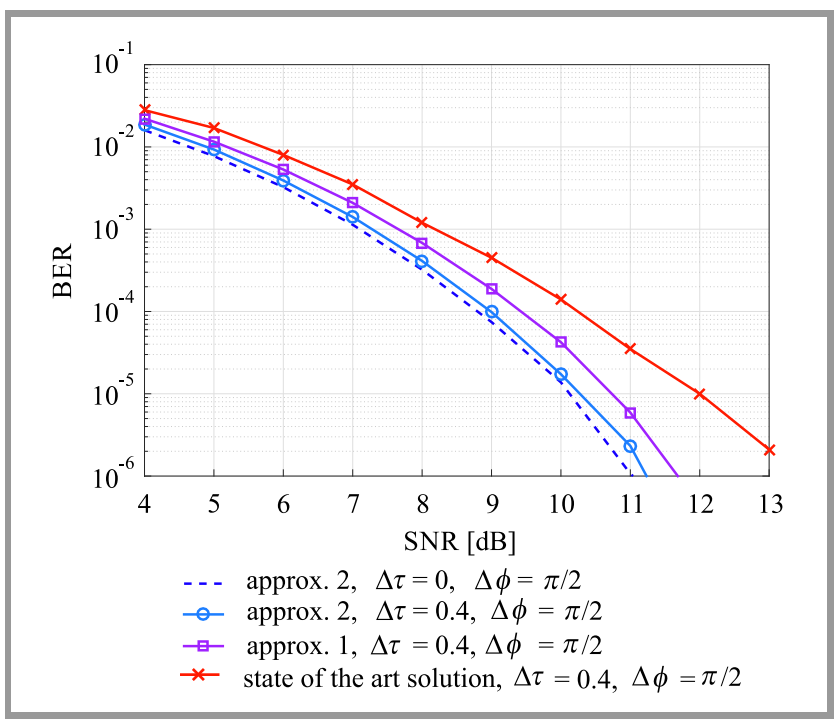

Fig. 5. BER performance for the different PAM approximations, $\Delta \tau=0.4, \Delta \phi=\frac{\pi}{2}$.

\subsection{BER Performance as a Function of $M$}

In Fig. 6, we plot the BER curves of the Viterbi $M$-algorithm for different values of $M$. One may notice that two best states instead of 16 taken at each epoch are sufficient to achieve nearly optimal performance. Therefore, incorporation of the $M$-algorithm for hard decoding may offer a considerable reduction in complexity, simultaneously maintaining very good BER performance.

\subsection{Coded Performance using M-SOVA}

Here, we evaluate the BER performance of the M-SOVA. To do so, bit stream $\underline{b}$ is encoded using a low density parity check (LDPC) encoder of rate $R=\frac{2}{3}$, with its number of bits contained in the information word $K$ being equal to 


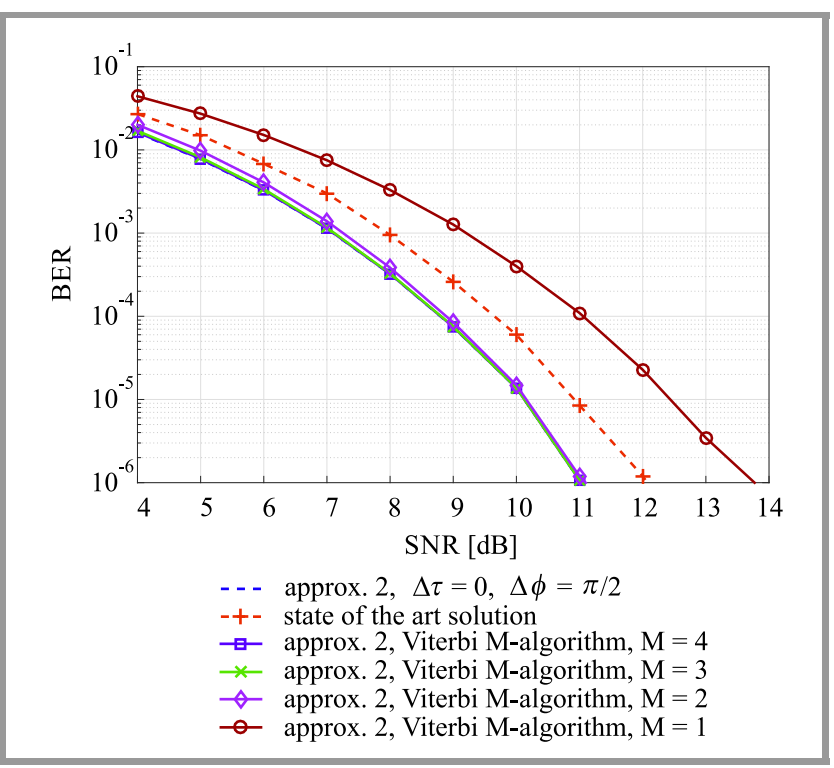

Fig. 6. BER performance for different $M$ values, $\Delta \tau=0, \Delta \phi=\frac{\pi}{2}$.

4,096. This encoder is developed at NASA's JPL and is fully described in [19]. It is also normalized in IRIG [1]. The LDPC-encoded bits are then Alamouti encoded as described in Section 2 to generate the two signals $s_{0}$ and $s_{1}$. The received signal is demodulated using the M-SOVA and the estimated LLRs are fed to the LDPC-decoder that performs a maximum of $N_{i t}=200$ iterations. The BER performance curves are plotted in Fig. 7 for different values

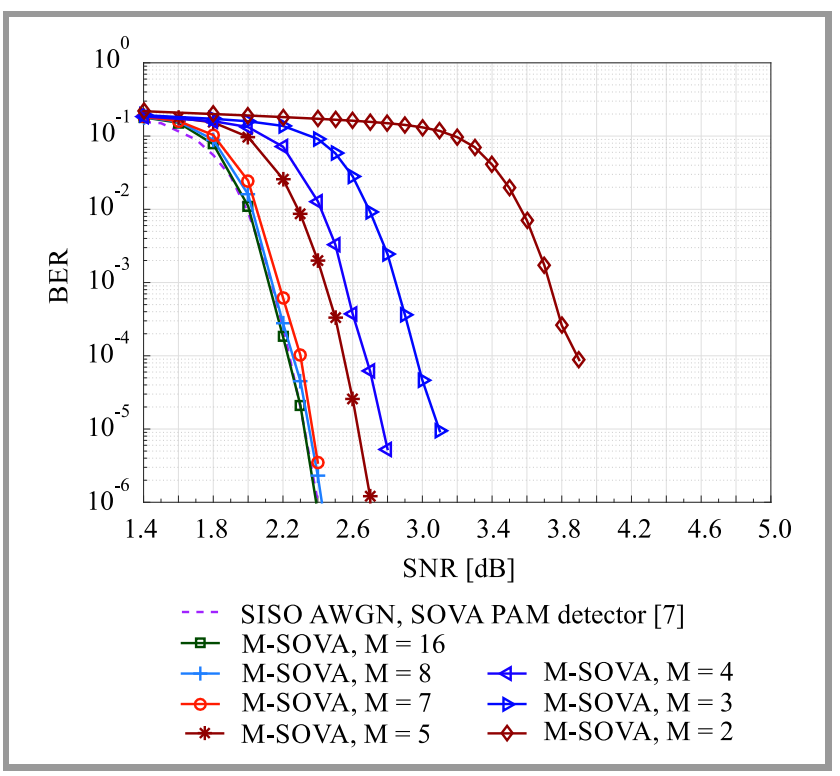

Fig. 7. BER performance for LDPC when paired with $M$-SOVA, $\Delta \tau=0, \Delta \phi=0$.

of $M$. The same figure shows also the BER performance of the SOVA detector for the single-input-single-output (SISO) case [7]. One may see that 5-SOVA only performs within $0.3 \mathrm{~dB}$ of the 16-SOVA case. Moreover, it may be seen that BER performance is more sensitive to $M$ when soft decoding is applied, since the LRR estimates increase as
$M$ is decreased. Despite that, the proposed $M$-SOVA offers a close BER performance when $M$ equals 5 .

\section{Conclusion}

In this paper, we presented a decoding architecture for Alamouti-encoded SOQPSK-TG signals. The proposed solution relies on the PAM approximation of SOQPSK-TG rather than on the XTCQM approximation and operates using a single trellis regardless of the sign of the differential delay between the two signals. We showed that soft outputs of the decoded bit stream may be extracted via the proposed M-SOVA while keeping a reasonable degree of complexity. Simulation results show that the proposed solutions offer better BER performance than the least squares XTCQM Alamouti decoder (state-of-the-art solution) when we consider the second PAM approximation for different channel configurations. It is also shown that LLRs provided via MSOVA offer very attractive BER performance when Alamouti encoding is combined with IRIG-normalized LDPC encoding.

\section{References}

[1] IRIG, "Telemetry Standards", IRIG Standard 106-17 Part 1, Chapter 2", July 2017 [Online]. Available: http://www.irig106.org/docs/ 106-17/106-17_Telemetry_Standards.pdf

[2] M. Rice, T. Nelson, J. Palmer, C. Lavin, and K. Temple, "Space-time coding for aeronautical telemetry: Part I - Estimators", IEEE Trans. on Aerosp. and Electron. Syst., vol. 53, no. 4, pp. 1709-1731, 2017 (doi: 10.1109/TAES.2017.2671784).

[3] M. A. Jensen, M. D. Rice, and A. L. Anderson, "Aeronautical telemetry using multiple-antenna transmitters", IEEE Trans. on Aerosp. and Electron. Syst., vol. 43, no. 1, pp. 262-272, 2007 (doi: 10.1109/TAES.2007.357132).

[4] S. M. Alamouti, "A simple transmit diversity technique for wireless communications", IEEE J. on Selec. Areas in Commun., vol. 16, no. 8, pp. 1451-1458, 1998 (doi: 10.1109/49.730453).

[5] M. Rice, T. Nelson, J. Palmer, C. Lavin, and K. Temple, "Space-time coding for aeronautical telemetry: Part II - Decoder and system performance", IEEE Trans. on Aerosp. and Electron. Syst., vol. 53, no. 4, pp. 1732-1754, 2017 (doi: 10.1109/TAES.2017.2671785).

[6] N. T. Nelson, "Space-time coding with offset modulations", Ph.D. Thesis, Department of Electrical and Computer Engineering, Brigham Young University - Provo, Dec. 2007.

[7] E. Perrins, "FEC systems for aeronautical telemetry", IEEE Trans. on Aerosp. and Electron. Syst., vol. 49, no. 4, pp. 2340-2352, 2013 (doi: 10.1109/TAES.2013.6621820).

[8] R. Othman, A. Skrzypczak, and Y. Louët, "PAM decomposition of ternary CPM with duobinary encoding", IEEE Trans. on Commun., vol. 65 , no. 10 , pp. 4274-4284, 2017 (doi: 10.1109/TCOMM.2017.2723567).

[9] T. Nelson, E. Perrins, and M. Rice, "Near optimal common detection techniques for shaped offset QPSK and Feher's QPSK", IEEE Trans. on Commun., vol. 56, no. 5, pp. 724-735, 2008 (doi: 10.1109/TCOMM.2008.060155).

[10] J. Tan and G. L. Stuber, "A MAP equivalent SOVA for non-binary turbo codes", in Proc. IEEE Int. Conf. on Commun. ICC 2000, New Orleans, LA, USA, 2000, vol. 2, pp. 602-606 (doi: 10.1109ICC.2000.853567).

[11] J. B. Anderson, "Instrumentable tree encoding of information sources", IEEE Trans. on Inform. Theory, vol. 17, no. 1, pp. 118-119, 1971 (doi: 10.1109/TIT.1971.1054572).

[12] K. K. Y. Wong, The Soft-Output M-Algorithm and Its Applications. Kingston, Ont., Canada: Queen's University, 2006 (ISBN: 978-0-494-18566-7). 
[13] J. Hagenauer and P. Hoeher, "A Viterbi Algorithm with soft-decision outputs and its applications", in Proc. IEEE Glob. Telecommun. Conf. and Exhib. "Communications Technology for the 1990s and Beyond", Dallas, TX, USA, 1989, vol. 3, pp. 1680-1686 (doi: 10.1109/GLOCOM.1989.64230).

[14] J. B. Anderson, T. Aulin, and C.-E. Sundberg, Digital Phase Modulation. New York: Plenum Press, 1986 (ISBN-13: 978-0306421952).

[15] T. Nelson and M. Rice, "A unified perspective on ARTM tier I waveforms - Part I: Common representations", in Proc. MILCOM 2005 - 2005 IEEE Milit. Commun. Conf., Atlantic City, NJ, USA, 2005, vol. 2, pp. 897-903 (doi: 10.1109/MILCOM.2005.1605794).

[16] E. Perrins and M. Rice, "PAM Representation of Ternary CPM", IEEE Trans. on Commun., vol. 56, no. 12, pp. 2020-2024, 2008 (doi: 10.1109/TCOMM.2008.041108).

[17] A. J. Viterbi, "An intuitive justification and a simplified implementation of the MAP decoder for convolutional codes", IEEE J. on Selec. Areas in Commun., vol. 16, no. 2, pp. 260-264, 1998 (doi: 10.1109/49.661114).

[18] V. Franz and J. B. Anderson, "Concatenated decoding with a reduced-search BCJR algorithm", IEEE J. on Selec. Areas in Commun., vol. 16, no. 2, pp. 186-195, 1998 (doi: 10.1109/49.661107).

[19] C. C. S. D. S, Low Density Parity Check Codes for Use in NearEarth and Deep Space Applications. Consultative Committee for Space Data Systems, 131.1-O-2 Orange Book, Sept. 2007 [Online]. Available: https://public.ccsds.org/Pubs/131x1o2e2s.pdf

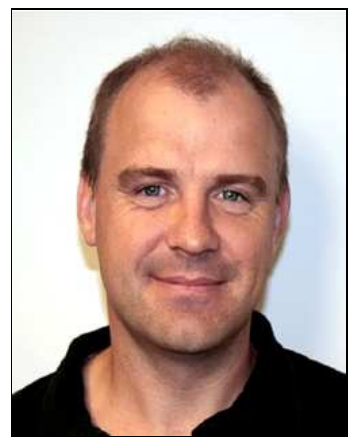

Yves Louët received his Ph.D. degree in Digital Communications from Rennes University, France, in 2000. His $\mathrm{Ph} . \mathrm{D}$. thesis focused on peakto-average power reduction in OFDM modulation with channel coding. In 2000 he assumed the position of a Research Engineer at Siradel, Rennes, and participated in channel propagation modeling for cell planning. In 2002, he became an Associate Professor at Supélec, Rennes. In his teaching and research activities, he focused on signal processing and digital communications used in software and cognitive radio systems. His research contributions deal primarily with new waveform design for green cognitive radio and with energy efficiency enhancements. In 2010, he received his research habilitation (HDR) from Rennes University and became a Professor with Supelec. Currently, he works at CentraleSupélec. $\mathrm{He}$ is the Head of the Signal Communication Embedded Electronics Research Group, a member of the Institute of Electronics and Telecommu- nications of the Rennes Lab, and a Chair of the URSI Commission $\mathrm{C}$.

E-mail: yves.louet@centralesupelec.fr

IETR/CentraleSupélec

Avenue de la Boulaie

35576 Cesson-Sévigné, France

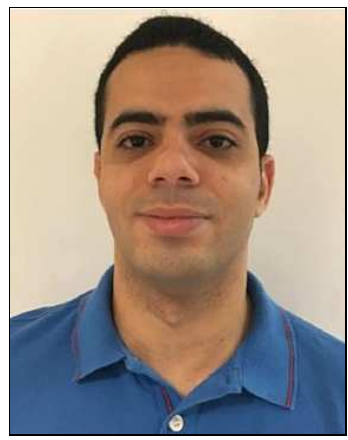

Rami Othman received his engineering degree in Digital Communications from Ecole Nationale de l'Aviation Civile, Toulouse, France, in 2015. He received his Ph.D. degree from the Signal, Communication, and Embedded Electronics Research Group, CentraleSupélec, Rennes, France in 2019.

E-mail: rami.othman@zodiacaerospace.com

Zodiac Data Systems

14460 Colombelles, France

IETR/CentraleSupélec

Avenue de la Boulaie

35576 Cesson-Sévigné, France

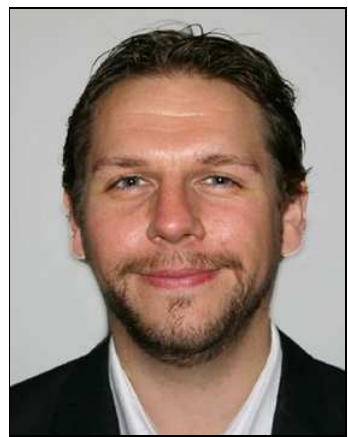

Alexandre Skrzypczak received his engineering degree in Telecommunications from Telecom Bretagne, France, in 2004, and Ph.D. degree in Signal Processing and Telecommunications from the University of Rennes, France, in 2007. After completing post-doctoral studies at Supélec Rennes, France, and focusing on academic career as a Researcher with ECAM Rennes, France, he joined Zodiac Data Systems in 2012 as a Research Engineer specializing in signal processing. His main research fields focus on multicarrier modulations and their robustness with respect to nonlinearities at the transmitter side, as well as on PAPR reduction, PA linearization and equalization of frequency modulations.

E-mail: alexandre.skrzypczak@zodiacaerospace.com Zodiac Data Systems

14460 Colombelles, France 